\title{
Additive Dosage Form Category
}

National Cancer Institute

\section{Source}

National Cancer Institute. Additive Dosage Form Category. NCI Thesaurus. Code

C149346.

A type of pharmaceutical dose form that may be liquid, semi-solid or solid, to be added to a large volume of diluent, such as in a bath. 